
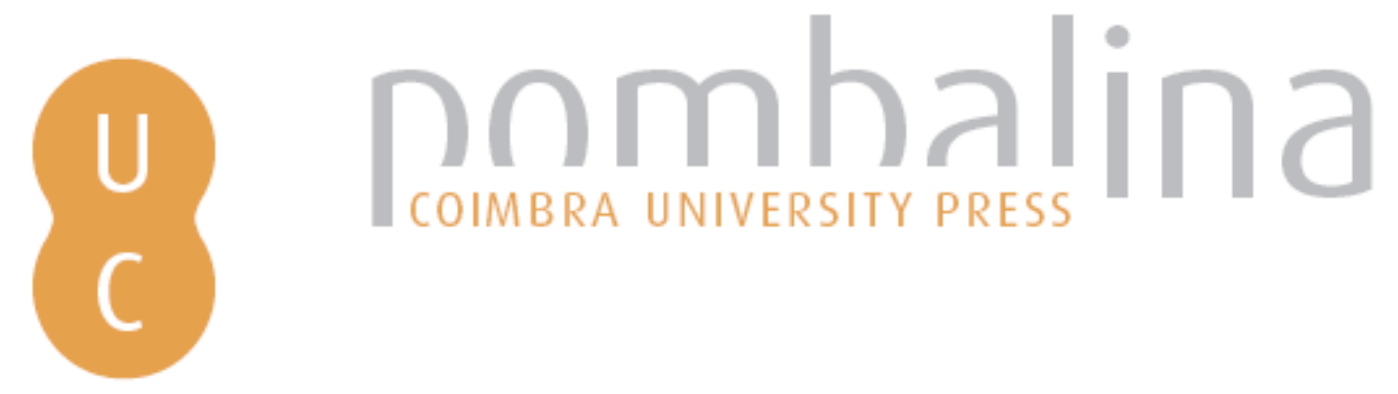

\title{
Produção automática de ortofotos em áreas urbanas utilizando veículos aéreos não- tripulados e software de código aberto
}

Autor(es): Duarte, Diogo; Gonçalves, Gil

Publicado por: Imprensa da Universidade de Coimbra

URL

persistente: URI:http://hdl.handle.net/10316.2/37095

DOI: $\quad$ DOI:http://dx.doi.org/10.14195/978-989-26-0983-6_42

Accessed : $\quad$ 26-Apr-2023 09:21:23

A navegação consulta e descarregamento dos títulos inseridos nas Bibliotecas Digitais UC Digitalis, UC Pombalina e UC Impactum, pressupõem a aceitação plena e sem reservas dos Termos e Condições de Uso destas Bibliotecas Digitais, disponíveis em https://digitalis.uc.pt/pt-pt/termos.

Conforme exposto nos referidos Termos e Condições de Uso, o descarregamento de títulos de acesso restrito requer uma licença válida de autorização devendo o utilizador aceder ao(s) documento(s) a partir de um endereço de IP da instituição detentora da supramencionada licença.

Ao utilizador é apenas permitido o descarregamento para uso pessoal, pelo que o emprego do(s) título(s) descarregado(s) para outro fim, designadamente comercial, carece de autorização do respetivo autor ou editor da obra.

Na medida em que todas as obras da UC Digitalis se encontram protegidas pelo Código do Direito de Autor e Direitos Conexos e demais legislação aplicável, toda a cópia, parcial ou total, deste documento, nos casos em que é legalmente admitida, deverá conter ou fazer-se acompanhar por este aviso.

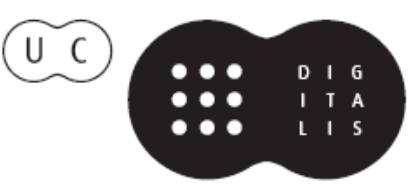




\section{$\forall$ \\ TAS DAS I JORNADAS LUSÓFONAS DE CIÊNCIAS E TECNOLOGIAS DE INFORMAÇÃO GEOGRÁFICA}

Editores

José Gomes dos Santos

Cidália Fonte

Rui Ferreira de Figueiredo

Alberto Cardoso

Gil Gonçalves

José Paulo Almeida

Sara Baptista 


\title{
ARTIGO 42
}

\section{PRODUÇÃo AUTOMÁtica de ORTOFOTOS EM ÁREAS URBANAS UTILIZANDO VEÍCULOS AÉREOS NÃO-TRIPULAdOS E SOFTWARE DE Código ABERTO}

DUARTE, Diogo ${ }^{1}$ e GONÇALVES, Gil1,2

\begin{abstract}
1 Departamento de Matemática-Faculdade de Ciências e Tecnologia da Universidade de Coimbra Apartado 3008; EC Universidade, 3001 - 454 Coimbra, Portugal; Tel: +351 239791150; Fax: +351 239793069; email: diogovad@gmail.com

2 Instituto de Engenharia de Sistemas e Computadores de Coimbra (INESC-Coimbra); Rua Antero de Quental, No199; 3000 - 033 Coimbra, Portugal; Tel: +351 239791150; Fax: +351 239793069; email: gil@ mat.uc.pt
\end{abstract}

\section{RESUMO}

Assistimos atualmente a uma crescente utilização fotogramétrica de Veículos Aéreos Não Tripulados (VANTs) em diversas aplicações, que vão desde a monitorização ambiental à agricultura e silvicultura de precisão. Um dos produtos mais frequentemente requisitados à Fotogrametria Digital são os ortofotos. De facto, ao possibilitarem a integração, em ambiente SIG, dos dados radiométricos e/ou espectrais contidos nas imagens ópticas com os dados geográficos e/ou cartográficos, os ortofotos permitem expandir as análises espaciais para outros tipos de atributos. Neste trabalho é descrita uma metodologia para a utilização de VANTS na produção automática de ortofotos. Utilizando software de código aberto e tendo por base um caso de estudo realizado na área urbana de Coimbra iremos analisar as potencialidades e os limites da utilização desta tecnologia no contexto da produção de ortofotos para actualização duma base de dados espacial municipal. Os resultados obtidos 
mostram que a utilização conjunto de VANTs com software fotogramétrico de código aberto permitem gerar ortofotos com uma excelente relação qualidade/preço.

\title{
PALAVRAS-CHAVE
}

Áreas urbanas, Fotogrametria digital, VANTs, Ortofotos, Software de código aberto.

\author{
AUTOMATIC PRODUCTION OF ORTOPHOTOS IN \\ URBAN AREAS USING UAVS AND OPEN SOURCE SOFTWARE
}

\begin{abstract}
Nowadays, the photogrammetric use of Unmanned Aerial Vehicles (UAVs) in several areas is a reality that grows from day to day in several market areas. In such areas, ranging from environmental monitoring to precision agriculture, the ortophotos are the most commonly requested digital photogrammetric product. In fact, by using a GIS platform, the ortophotos can enable the integration of radiometric and spectral image data with the geographic and cartographic data and allow us to expand the spatial analysis to other types of attribute data. In this paper we present a methodology for the automatic production of ortophotos in urban areas by using UAVs and open source photogrammetric software. The potential and limits of this technology are assessed in the context of updating a municipal spatial database. The results show that the synergistic use of UAVs and open source photogrammetric software can be effectively used to produce ortophototos of small urban areas with an excellent quality/price ratio.
\end{abstract}

\section{KEYWORDS}

Urban areas, Digital photogrammetry, UAVs, Ortophotos, Open source software.

\section{INTRODUÇÃO}

A vulgarização da ortofotografia, que até há muito pouco tempo, estava directamente conotada com elevados custos, tanto económicos como de processamento, é uma realidade inquestionável devido à tremenda evolução tecnológica das câmaras digitais, do poder de cálculo e armazenamento dos computadores pessoais e à acessibilidade generalizadas aos VANTs. Actualmente, esta tecnologia permite um acesso generalizado da comunidade fotogramétrica e não fotogramétrica às técnicas de modelação tridimensional (3D) da superfície topográfica, as quais se baseiam num conjunto de imagens dessa superfície (ou objecto) e que obedecem 
a determinados protocolos intrínsecos à fotogrametria. Hoje em dia, com a ajuda dos VANTs e da grande comunidade que desenvolve ferramentas de código aberto, existe a possibilidade de tornar a realização de ortofotos uma tarefa mais agilizada e utilizá-la num maior leque de aplicações onde num passado recente não faria sentido devido aos seus elevados custos e à sua morosidade. A capacidade de intervenções rápidas e/ou sistemáticas, tornam os VANT a vanguarda de muitas operações, como por exemplo a análise de cenários de catástrofe ou a agricultura de precisão.

Em meio urbano e para apoio de várias actividades municipais é prática vulgar a utilização de ortofotos como camada básica de suporte a bases de dados espaciais (Skarlatos, 1999). De facto, ao possibilitarem a integração, em ambiente SIG, dos dados radiométricos e/ou espectrais contidos nas imagens ópticas com os dados geográficos e/ou cartográficos, os ortofotos permitem expandir as análises espaciais para outros tipos de atributos (Zhou et al., 2005).

Neste artigo iremos avaliar as potencialidades dos VANTs e do software de código aberto na produção de ortofotos em meio urbano. Na secção seguinte iremos apresentar a tecnologia VANTS e caracterizar a plataforma aérea utilizada. De seguida descreveremos os principais módulos do software de código aberto de fotogrametria digital utilizado na produção do ortofoto: o PAMP. A avaliação das potencialidades desta tecnologia é feita depois quer em termos quantitativos, quer em termos qualitativos. Para avaliar quantitativamente as potencialidades desta tecnologia iremos utilizar dois dos produtos gerados, o ortomosaico (ou ortofoto) e o Modelo Digital de Superfície (MDS) e iremos calcular a raiz quadrada do erro médio quadrático utilizando pontos de verificação obtidos pela tecnologia GNSS em modo RTK. Na avaliação qualitativa iremos comparar visualmente o ortomosaico gerado por esta tecnologia e outro gerado por um pacote de fotogrametria digital, o Pix $4 \mathrm{D}^{\circledR}$.

\section{VEÍCULOS AÉREOS NÃO-TRIPULADOS}

Em geral os VANT foram sempre associados a actividades militares (Watts et al., 2012). Recentemente, a comunidade civil têm vindo a 
utilizá-los num grande leque de aplicações científicas e não-científicas. Para além do factor económico, os VANT abrem um conjunto do novas possibilidades. Em muitos casos e devido à sua alta mobilidade e baixo custo podem introduzir a componente tempo em projectos onde as alterações que ocorrem no objecto são consideravelmente rápidas e onde o recurso a plataformas tradicionais seria incomportável do ponto de vista económico. Além disso nem todos os projectos precisam da elevada exactidão e precisão decorrente de uma plataforma aérea tradicional, apesar de, em determinadas situações, se poderem atingir níveis de exactidão semelhantes à dos voos tradicionais (Turner et al., 2012). Uma das principais desvantagens existentes actualmente em Portugal na utilização dos VANTS para actividades fotogramétricas reside na falta de legislação que regulamente o sua utilização. No entanto, o seu uso tem crescido rapidamente, o que obrigará, num futuro próximo, à criação de regulamentos e de fiscalização próprias pelas autoridades competentes. Outra desvantagem técnica importante, reside na deficiente exactidão das estimativa automática da orientação externa das imagens, dado que os VANT não são tão estáveis nem contêm unidades de medição inercial tão precisas como as utilizadas nas plataformas tradicionais (Küng et al., 2011). Na maior parte dos projectos é necessário a realização de ortofotos havendo apenas como dados entrada as fotos realizadas e apoio GNSS ( usualmente em RTK).

Para uma melhor compreensão comparemos a obtenção de imagens recorrendo a plataformas tradicionais com a obtenção de imagens através de VANT. A diferença de alturas de voo é significativa. A sobreposição deve ser maior nos VANTs, devido ao processo de obtenção automática de pontos de ligação assim como à fraca determinação das posições das câmaras e da sua atitude. Esta sobreposição, apresenta, geralmente uma maior variação, de par para par de fotos, no seu valor, relativamente às plataformas tradicionais. Outra diferença são as elevadas distorções de perspectiva devido principalmente à grande variação de alturas relativamente à altura de voo realizada. Os parâmetros de orientação externa tornam-se deste modo, desconhecidos, ou, quando medidos, não tenham 
a exactidão desejada, caso que não acontece nas plataformas tradicionais onde existem unidades de medição inercial, câmaras e receptores GNSS de alta qualidade onde nem o espaço nem o peso do material é relevante (Küng et al., 2011). Este último facto é de relativa importância, tendo em conta que o ajustamento por feixe de um bloco de fotografias necessita de uma boa aproximação inicial para calcular os parâmetros de orientação externa, podendo não convergir tal não aconteça. É usual, devido ao seu peso e dimensões, usar câmaras fotográficas amadoras de pequeno e médio formato. As imagens obtidas por VANTs apresentam também grandes variações tanto radiométricas como de resolução o que afecta também a qualidade final de um ortomosaico, factor que deve ser tido em consideração aquando da realização do processamento das imagens.

\section{1. mini-VANT Swinglet CAM}

Este VANT é composto por uma asa eléctrica (tipo delta) com um peso de 500g, incluindo o piloto automático e a câmara digital de 12 MPix (ver Figura 1). O seu baixo peso combinado com a fuselagem em espuma, torna-o relativamente seguro em aplicações urbanas, dado que a sua energia de impacto é equivalente à de uma ave de tamanho médio (Küng et al., 2011). Por outro lado, a sua baixa velocidade-ar (aprox. 36 $\mathrm{km} / \mathrm{h}$ ), o facto da sua descolagem ser feita por lançamento manual e a aterragem ser feita em espirais apertadas e em espaços relativamente curtos, facilitam ainda o planeamento do voo em zonas urbanas.

O swinglet CAM vem equipado do software eMotion (senseFly, 2014) que controla o piloto automático a partir dum computador portátil localizado em terra. Além disso permite também efectuar o planeamento da cobertura fotográfica e injectar a trajetória na memória do VANT juntamente com a localização das exposições. 


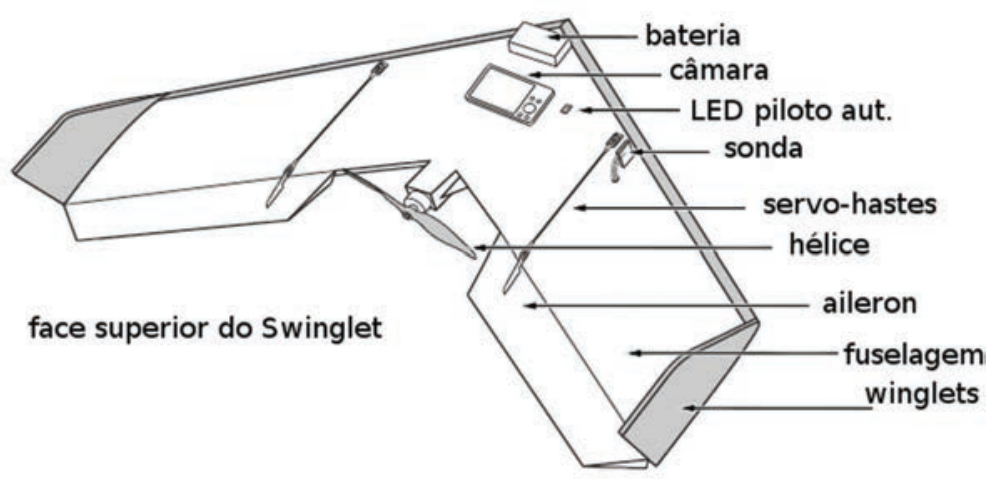

Figura 1 - O VANT utilizado neste estudo. Adaptado de (Sensefly, 2014)

\section{PAMP: SOFTWARE de Código ABERTo EM Fotogrametria digital}

Os pacotes de programas de código aberto são hoje em dia amplamente utilizados em todas as áreas científicas, devido tanto ao factor económico como à transparência dos métodos e algoritmos que são utilizados. Sendo o factor económico, hoje mais que nunca, importante, o controlo e a possibilidade de consultar o que o algoritmo faz e como o faz assume uma importância relevante, principalmente quando se tentam conhecer e quantificar as fontes de erro que podem afectar os outputs do software. Há ainda a salientar o facto de que muitas vezes o que acontece é a compra de licenças de código aberto por parte de empresas fabricantes de software para as poderem usar comercialmente, criando apenas interfaces gráficas que facilitam o acesso ao utilizador.

O PAMP (Pastis/Apero/Micmac/Porto) é um pacote de programas de código aberto criado por Marc Pierrot-Deseilligny do Instituto Geográfico Francês. Inicialmente continha apenas ferramentas para a correspondência de imagens. No entanto, foram integradas outras ferramentas já existentes, podendo actualmente realizar a maioria das tarefas, a montante e a jusante da correspondência de imagens com vista à modelação 3D de objectos a partir dum conjunto de imagens. Este pacote é escrito em C++ e contém várias ferramentas para: 
- extracção de modelos digitais de superfície a partir de múltiplos pares estereo,

- modelação tridimensional de superfícies/objectos,

- registo de imagens multi-espectrais

- rectificação de imagens satélite utilizando coeficientes polinomiais racionais RPC's

- produção de ortofotos

Este software recorre, por sua vez aos seguintes pacotes (também de código aberto), nomeadamente: i) o SIFT (Scale Invariant Feature Transform) para a identificação de pontos homólogos entre imagens; ii) o DCRAW para extrair informação exif das imagens; iii) o Image Magick para a manipulação e conversão entre diferentes formatos de imagens; iv) o proj4 para a transformação entre diferentes sistemas de coordenadas.
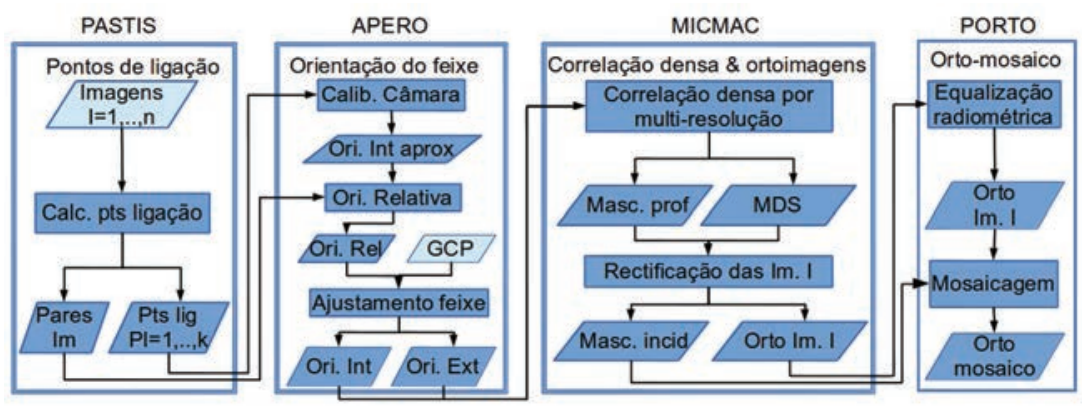

Figura 2 - Pipeline seguida para a geração automática do orto e do MDS

O PAMP é composto por 4 módulos (ver Figura 2): i) PASTIS para a determinação dos pontos de ligação entre as várias imagens do bloco; ii) APERO para o ajustamento por feixe do bloco de fotografias; iii) MicMac para a correlação densa entre múltiplos pares estéreo e produção das ortimagens; iv) PORTO para a mosaicagem e equalização radiométrica das ortoimagens e a consequente produção do ortofoto da cobertura.

\subsection{PASTIS}

A primeira etapa do processo de construção do ortofoto consiste na determinação dos pontos de ligação entre as imagens que constituem a cobertura aérea e na consequente ordenação das imagens através da 
formação dos possíveis pares estereo (ver Figura 2). Esta tarefa é feita com o PASTIS (Programme utilisant Autopano Sift pour les Tie-points dans les ImageS). Numa primeira fase são determinados os pontos de interesse utilizando o detector SIFT os quais são supostamente robustos relativamente às variações de escala, às rotações e ao ruído. Numa segunda fase os pontos de interesse são emparelhados, ou seja, para cada imagem e para cada ponto são determinadas as imagens que contêm um ponto semelhante, definido segundo a norma euclidiana (Lowe, 2004).

\subsection{APERO}

Depois de termos determinado os pontos de ligação entre as imagens passamos à orientação do feixe de imagens num determinado referencial definido pelo utilizador. Esta etapa do processo é feita com o APERO (Aérotriangulation Photogrammétrique Expérimentale Relativement Opérationnelle). Os principais módulos do APERO são (Pierrot-Deseilligny and Clery, 2011):

- Um módulo para o cálculo da aproximação inicial da orientação do bloco, que recorre a: i) um algoritmo baseado na matriz essencial duma projecção cónica, utilizada vulgarmente na visão computacional; ii) à recessão espacial fotogramétrica (i.e orientação externa); iii) a um programador especial hierárquico que determinar a árvore óptima do bloco das fotografias (onde cada imagem com excepção da primeira tem um conjunto de pais relativamente à qual a sua orientação é calculada)

- Um módulo para o cálculo do ajustamento por feixe, baseado na linearização clássica das equações de colinearidade e no consequente processo iterativo de Gauss-Newton ou em opção o método de Levenberg-Marquardt para a resolução do sistema de equações não-lineares.

- Módulos para a determinação da orientação absoluta baseada em pontos de apoio ou definida a partir das imagens.

- Alguns módulos para a importação e exportação de dados: pontos de ligação, pontos de apoio, orientações interna e externa, coordenadas GPS dos centros de exposição (no caso de existirem). 


\subsection{MICMAC}

A etapa seguinte do processo consiste na geração duma nuvem de pontos 3D utilizando a correspondência densa de imagens. No MicMac (Multi-Images Correspondances, Méthodes Automatiques de Corrélation) o problema de reconstrução da superfície por correlação densa é resolvido pela minimização dum funcional de energia dado por (Pierrot-Deseilligny and Paparoditis, 2006):

$$
E_{\alpha}(Z(x, y))=\sum(1-\operatorname{Corr}(x, y, Z(x, y)))+\alpha K(x, y)
$$

Nesta equação $Z(x, y)$ é a superfície que pretendemos reconstruir (isto é o MDS), Corr é a função de correlação cruzada normalizada, ALFA é um parâmetro de suavização e $K$ é o termo de regularização dado por

$$
K(x, y)=|Z(x+1, y)-Z(x, y)|+\mid Z(x, y+1)-Z(x, y)
$$

O mínimo global do funcional é encontrado em tempo polinomial utilizando uma implementação, em multi-resolução, da técnica clássica de optimização combinatória baseada no corte mínimo e no fluxo máximo de grafos (Roy and Cox, 1998).

\subsection{PORTO}

O Porto é a ferramenta utilizada para a produção de ortofotos (mosaicos de ortoimagens). Apesar de ainda estar num estado muito incipiente de desenvolvimento (Pierrot-Deseilligny, 2012) permite já realizar a mosaicagem e a equalização radiométrica das diferentes orto-imagens que constituem o bloco. A equalização radiométrica de cada $O_{i}(x, y)$ ortoimagem é feita através dum polinómio local $\mathrm{Pi}(x, y)$ e dum polinómio global $R(x, y)$ de forma a evitar uma possível deriva da radiometria:

$$
O_{\text {corr }}=O_{i}(x, y) P_{i}(x, y) R_{i}(x, y)
$$

Os coeficientes destes polinómios são determinados globalmente utilizando o método dos mínimos quadrados. 


\subsection{Parametrização formal da PAMP em XML}

Do ponto de vista prático, um dos inconvenientes do pacote PAMP reside na parametrização formal em XML dos seus diferentes módulos. Como o seu desenvolvimento foi feito tendo em vista um grande leque de aplicações aéreas e terrestres a parametrização é complexa. No entanto, existe para cada módulo um ficheiro xml padrão que poderá ser costumizado em função das especificidades de cada projecto.

No Pastis a informação necessária a incluir no ficheiro xml refere-se aos parâmetros iniciais de calibração da câmara, nos casos em que alguma desta informação não estiver contida na informação exif dos ficheiros imagem, isto é, as coordenadas imagem do ponto principal, a distância focal e o tamanho do sensor.

No caso do Apero esta parametrização em xml permite controlar os procedimentos de calibração da câmara e de orientação das imagens. Note-se que em muitos projectos fotogramétricos, este aspecto é muito importante pois é frequente ter alguns parâmetros de calibração/orientação previamente determinados e se pretende que estes se mantenham fixos durante o processo de refinamento dos parâmetros. Assim, as principais secções do ficheiro xml são:

- <SectionBDD_Observation > - onde se define os pontos de apoio e de ligação da cobertura,

- <SectionInconnues> - onde se lista a calibração da câmara (ou das câmaras) utilizadas no projecto e as imagens associadas. A primeira imagem da lista é posicionada de forma arbitrária,

- <SectionSolveur> - onde se especifica qual o algoritmo a utilizar na resolução da triangulação fotogramétrica,

- <SectionCompensation> - onde se definem as diferentes etapas do cálculo da triangulação.

No caso do MicMac a parametrização xml é em geral utilizada em projectos de fotogrametria terrestre, para se adaptar a geometria da rede de imagens na reconstrução 3D do objecto de estudo. É também neste 
ficheiro $x m l$ que se define espacialmente a área de trabalho e se parametrizam as várias etapas de multi-resolução referidas anteriormente. As secções mais importantes são:

- <Section_Terrain> - onde é definida a área sobre a qual o MicMac vai realizar a correspondência entre imagens, assim como o intervalo de profundidade

- <Section_PriseDeVue> - onde se definem as vários perspectivas de imageamento do objecto, com as suas imagens e parâmetros.

- <Section_MEC> - é onde se define as várias etapas multi-resolução e os seus respectivos parâmetros (zoom, janela de correlação, ...)

Por último a informação mais importante a considerar no ficheiro xml do Porto é relativa à montagem do mosaico (<SectionFilatrageIn>) e à aplicação das correcções radiométricas (<SectionEgalisation $>$ ) pretendidas. Tal como no MicMac a parametrização xml do Porto é feita em geral em projectos de fotogrametria terrestre, devido à possibilidade de existirem diferentes perspectivas de imageamento do objecto.

\section{CASO DE ESTUDO: ZONA URBANA DE COIMBRA}

\subsection{Dados}

O caso de estudo apresentado neste trabalho situa-se na zona central da cidade de Coimbra, cobrindo uma área urbana, a Praça da República, e uma área de vegetação, o Parque de Santa Cruz (Figura 3). O campo de jogos do Parque serviu como zona de descolagem e de aterragem do VANT.

O voo foi efectuado no dia 28 de Janeiro de 2013 e consta dum bloco de 76 imagens obtidas com o swinglet CAM da senseFly (ver Figura-4). A recolha dos pontos de apoio (GCPs) e dos pontos de verificação (CHP) foi feita por técnicas GNSS em modo RTK (ver Figura 3). O planeamento de voo foi feito com o software eMotion com os requisitos de $80 \%$ de sobreposição longitudinal, 60\% de sobreposição lateral e uma altura de voo média de 100 (aproximadamente) acima do solo urbano. A localização dos centros de exposição é também ilustrada na Figura 3. 


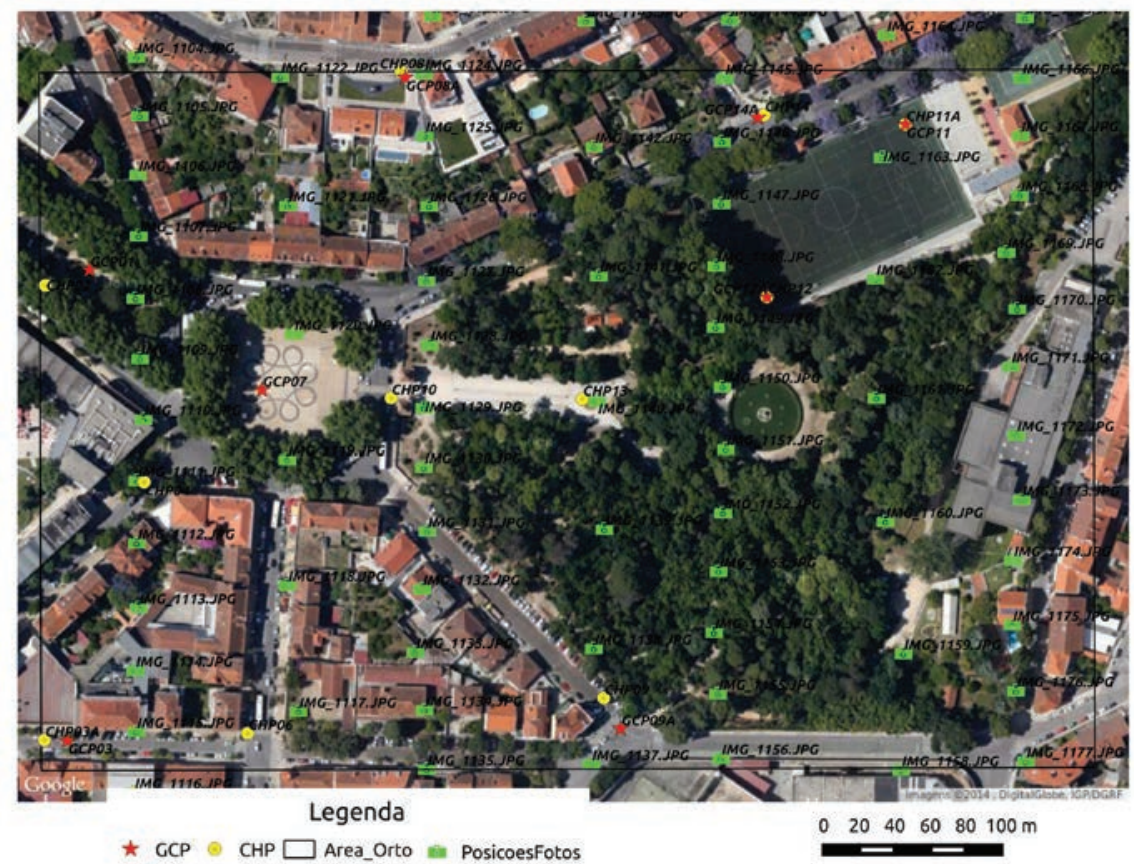

Figura 3 - Localização da zona de estudo, dos centros das imagens e dos pontos de apoio e verificação
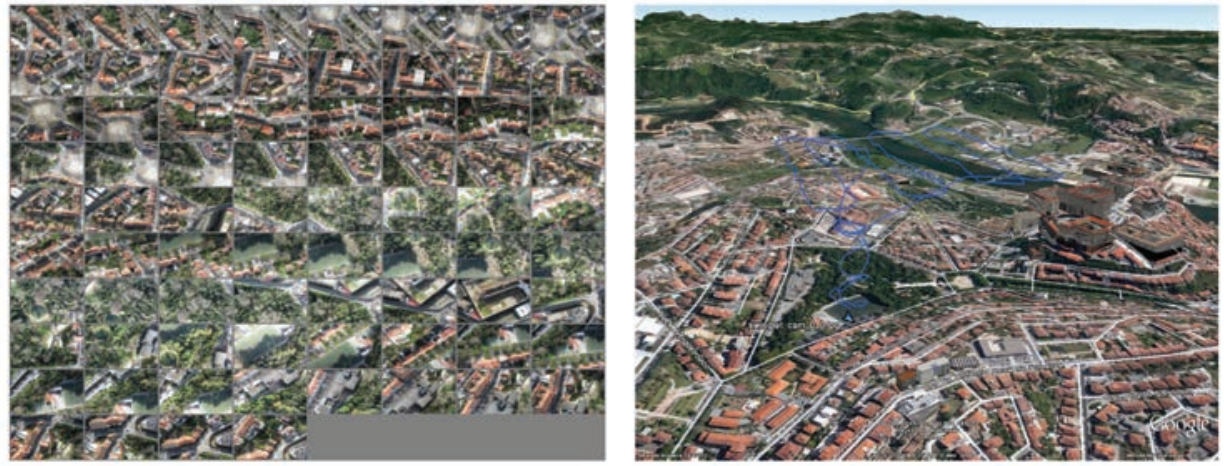

Figura 4 - Bloco de 76 imagens que compõem a cobertura aérea da área de estudo e correspondente trajectória. A vermelho imagens utilizadas na calibração inicial da câmara

\subsection{Resultados e Discussão}

A Figura 5 mostra o ortofoto da cobertura aérea obtido pelo pacote de software fotogramétrico de código aberto PAMP, utilizando a configuração 
descrita seguidamente. Para a calibração inicial da câmara utilizaram-se 14 imagens do bloco (Figura 4) e o modelo de distorção radial das lentes estendido definido com os 5 parâmetros (Cx, Cy, R3, R5, R7), além da posição do ponto principal (Pierrot-Deseilligny, 2012). Para apoio da triangulação do bloco das 76 imagens no sistema de coordenadas PT-TM06/ ETRS89 utilizaram-se 11 pontos de apoio (Figura 3). para a normalização radiométrica das ortoimagens.

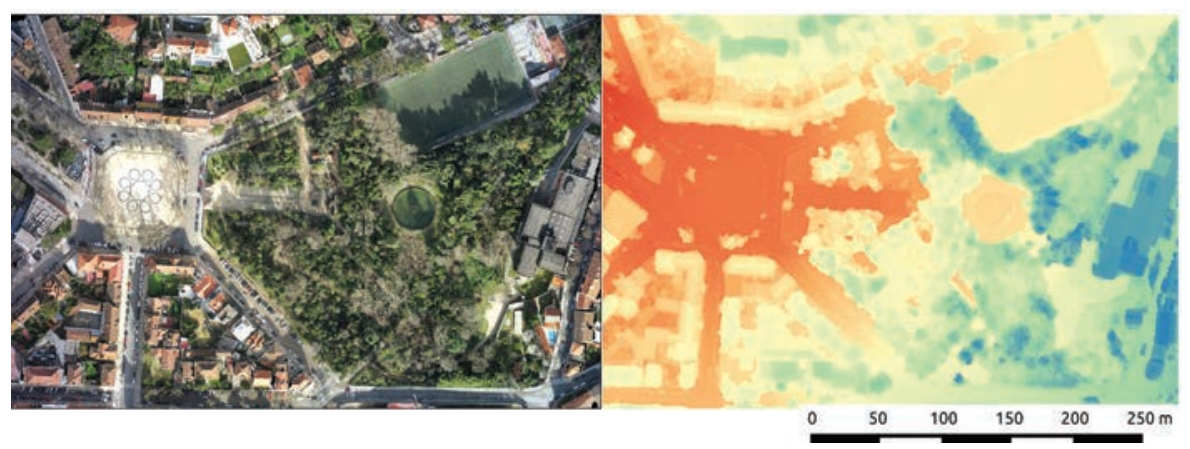

Figura 5 - Ortofoto da cobertura e correspondente MDS obtido per correlação densa (ambos com resolução espacial de $4 \mathrm{~cm}$ )

A Figura 6 mostra uma perspectiva da nuvem de pontos 3D obtida pelo software de código aberto PAMP e colorida pelos valores RBG das 76 imagens da cobertura. Esta nuvem é composta por 136296981 pontos e ocupa um volume aproximado de 2,0GB.

A Tabela 1 mostra os resultados obtidos na avaliação da extactidão posicional do orto e na avaliação da exactidão vertical do MDS. No cálculo da tabela consideraram-se as seguintes expressões:

$$
\Delta=\mathrm{C}_{\mathrm{GPS}}-\mathrm{C}_{\text {Orto }} ; \quad \text { remq }=\sqrt{\frac{\left((\Delta M)^{2}+(\Delta P)^{2}\right)}{n}}=\sqrt{\mathrm{remq}_{M}^{2}+\mathrm{remq}_{P}^{2}}
$$

Os valores obtidos mostram que a exactidão posicional do orto avaliada pelo indicador da raiz quadrada do erro médio quadrático do orto é de $19 \mathrm{~cm}$ a qual se mostra perfeitamente adequada para actividades municipais. 


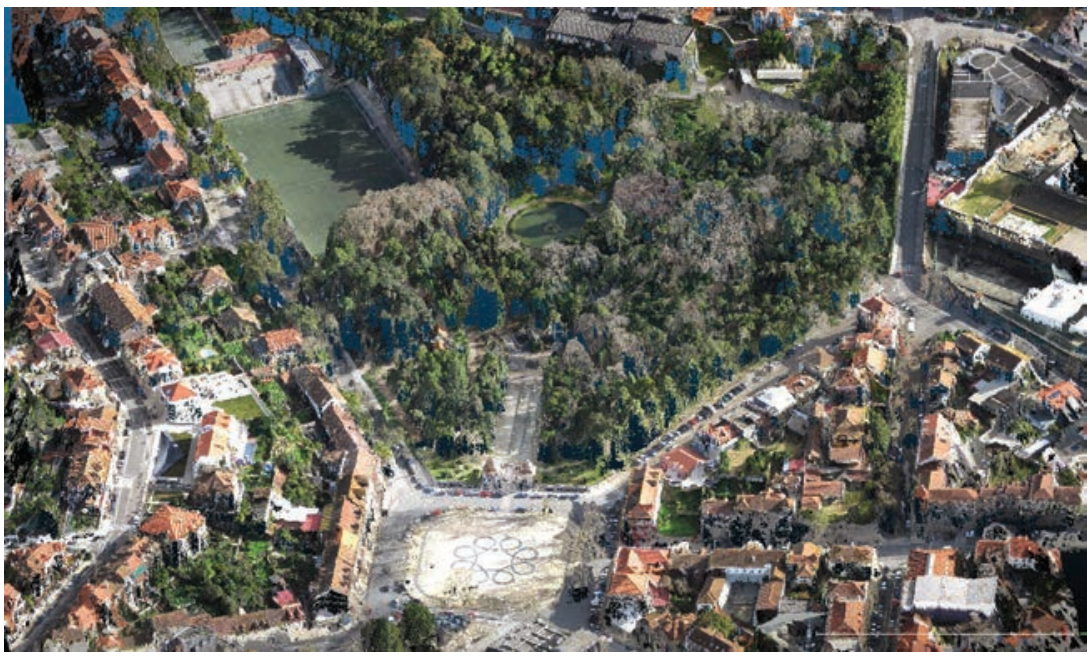

Figura 6 - Nuvem de pontos 3D contendo 136296981 pontos

Para avaliarmos a influência do número dos pontos de apoio (GCPs) na triangulação por feixe repetiu-se o procedimento anterior mas considerando desta vez apenas os 4 pontos GCPs seguintes: 07, 08A, 09A, 11. Os resultados globais obtidos foram praticamente idênticos aos da tabela com a excepção do rmeq_M que foi superior em apenas $1 \mathrm{~cm}$.

Tabela 1 - Avaliação da exactidão posicional do ortofoto e vertical do MDS

\begin{tabular}{|c|c|c|c|c|}
\hline \multirow{2}{*}{$\begin{array}{c}\text { CHP } \\
\mathbf{N}^{\circ} \mathbf{P T}\end{array}$} & \multicolumn{3}{|c|}{ Resíduos Orto (m) } & \multirow{2}{*}{$\begin{array}{c}\text { MDS } \\
\Delta \mathrm{H}(\mathrm{m})\end{array}$} \\
\hline & $\Delta \mathbf{M}$ & $\Delta \mathbf{P}$ & Erro_xy & \\
\hline 02 & 0,109 & 0,048 & 0,119 & $-0,774$ \\
\hline 04 & 0,051 & 0,129 & 0,139 & 0,299 \\
\hline 06 & $-0,023$ & 0,008 & 0,024 & $-0,152$ \\
\hline 08 & $-0,128$ & 0,221 & 0,255 & $-0,144$ \\
\hline 09 & 0,058 & $-0,057$ & 0,081 & 0,195 \\
\hline 10 & $-0,069$ & 0,013 & 0,070 & 1,353 \\
\hline $11 \mathrm{~A}$ & $-0,113$ & $-0,01$ & 0,113 & $-0,799$ \\
\hline 12 & $-0,082$ & $-0,005$ & 0,082 & 0,341 \\
\hline 14 & $-0,19$ & $-0,026$ & 0,192 & 0,054 \\
\hline
\end{tabular}

\subsection{Comparação relativa}

Afim de testarmos a performance deste software de código aberto num 
contexto de produção, surgiu a ideia de se efectuar uma comparação relativa entre o orto gerado nas secções anteriores e orto gerado pelo software comercial Pix4D®. A Figura 7 mostra uma comparação relativa visual entre estes dois ortos. Na Figura 7 -a é apresentado o orto produzido pelo Pix4D®. Na Figura 7 -b é apresentado a diferença RGB entre este orto e o produzido pelo PAMP (Figura 5 -a); note-se que a cor cinzenta representa os pixeis com valores semelhantes de RGB. Nas Figura 7 -c e 7-d é apresentado o mesmo detalhe no orto gerado pelo PAMP e pelo Pix4D, respectivamente. O mesmo acontece nas Figuras 7-e e 7-f.

Apesar de globalmente a qualidade radiométrica dos dois ortos ser muito semelhante existem pequenas diferenças que interessa salientar: i) no PAMP existem alguns pixeis sem valor RGB (pixeis a preto); ii) no PIX4D apesar de não existirem pixeis pretos alguns deles são substituídos por valores incorrectos de RGB originando os chamados pixeis fantasma.

\section{CONCLUSÕES}

Pode-se dizer que os VANT não vão tornar as técnicas da fotogrametria aérea clássica obsoletas. Antes pelo contrário, vêm abrir um novo leque de oportunidades na área da Fotogrametria Digital, trazendo consigo a necessidade de desenvolver e utilizar novos pacotes de software fotogramétrico capazes de lidar tanto com a quantidade de informação obtida, assim como a de ultrapassar a utilização de imagens obtidas por câmaras de grande consumo e o facto destas imagens serem captadas em posições e atitudes não convencionais necessárias à maioria dos programas clássicos de aerotriangulação por feixe de blocos de imagens.

Os resultados obtidos para os indicadores de extactidão posicional do orto (remq_orto $=11 \mathrm{~cm}$ ) mostram que os VANTs conjugados com o software de código aberto são uma tecnologia a considerar seriamente para a produção de ortofotos em meio urbano pelas autarquias. No entanto a presença de alguns artefactos (pixeis sem valor de RGB ou pixeis fantasmas) pode ainda constituir um obstáculo para potenciais utilizadores.

Por outro lado, os resultados obtidos para os indicadores de exactidão vertical do MDS (remq_MDS $=60 \mathrm{~cm}$ ) sugerem que esta tecnologia poderá ser utilizada com alguma fiabilidade na geração de produtos $3 \mathrm{D}$, como por exemplo nuvens de pontos $3 \mathrm{D}$, e constituir uma fonte dados 
economicamente viável para variados fins, tais como o cadastro predial 3D.

Por outro lado, os resultados obtidos para os indicadores de exactidão vertical do MDS (remq_MDS $=60 \mathrm{~cm}$ ) sugerem que esta tecnologia poderá ser utilizada com alguma fiabilidade na geração de produtos 3D, como por exemplo nuvens de pontos $3 \mathrm{D}$, e constituir uma fonte dados economicamente viável para variados fins, tais como o cadastro predial 3D.

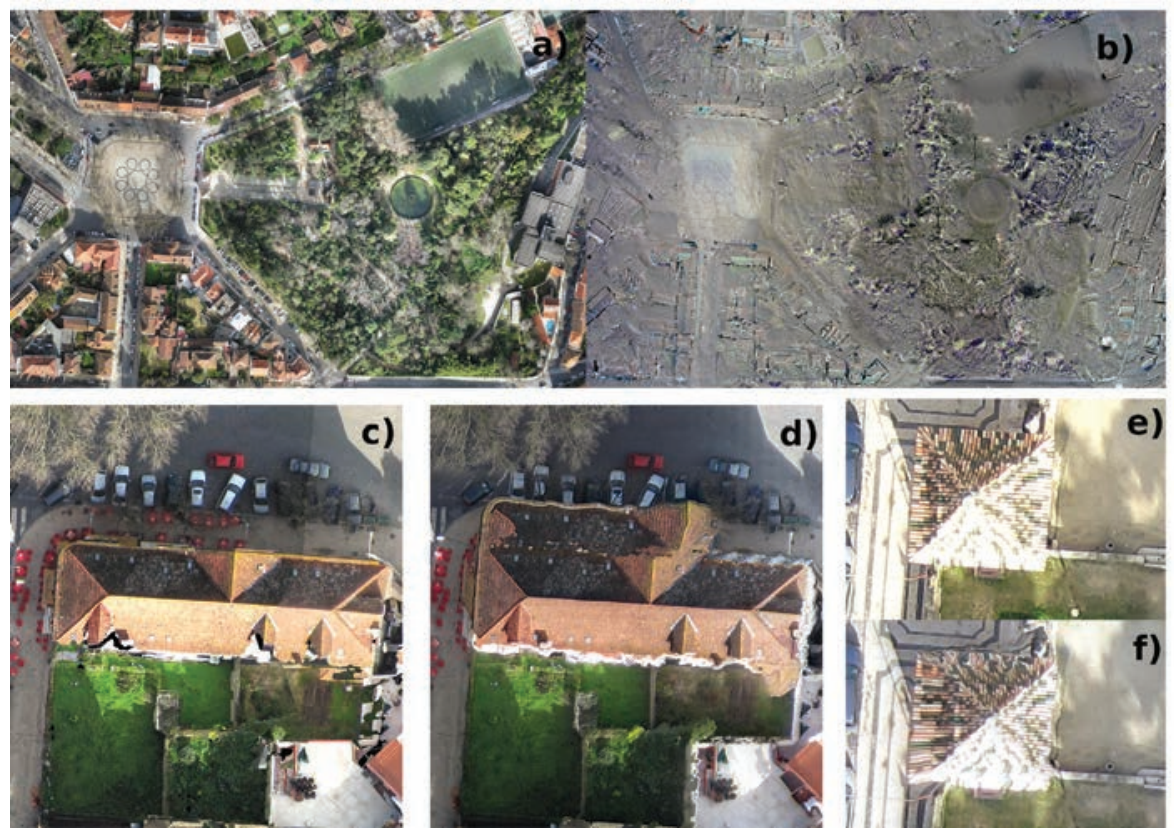

Figura 7 - Comparação visual entre os dois ortofotos: a) ortofoto produzido pelo Pix4D®; b) diferença radiométrica RGB pixel a pixel entre os dois ortofotos; c-e) e d-f) representam, respectivamente, detalhes dos ortofotos produzidos pelo PAMP e pelo Pix4D

No entanto, atendendo a que PAMP, é um pacote de software que pretende abranger um grande leque de tarefas, requer também uma grande parametrização (ficheiros.$x m l$ ), o que aliado à não existência de um interface gráfico torna a sua utilização pouco amigável e algo complexa. A realização duma interface gráfica dentro dum ambiente SIG de código aberto (por exemplo o QGIS ou o GRASS) será alvo de futuro desenvolvimento. 


\section{AGRADECIMENTOS}

Os autores agradecem à SINFIC pela cedência das imagens e pelo orto gerado no software $\mathrm{Pix} 4 \mathrm{D}{ }^{\circledR}$. Agradecem também à Câmara Municipal de Coimbra pelos trabalhos de campo efectuados na recolha dos pontos de controlo (GCP) e dos pontos de validação (CHP). Por último salientam o facto deste trabalho ser financiado parcialmente pela Fundação para a Ciência e Tecnologia (FCT) no âmbito do projecto PEst-OE/ EEI/UI308/2014 e pelo Projecto EMSURE - Energy and Mobility for Sustainable Regions (CENTRO-07-0224-FEDER-002004) da iniciativa Energy for Sustainability da Universidade de Coimbra.

\section{BIBLIOGRAFIA}

KÜNG, O., Strecha, C., Beyeler, A., Zufferey, J.-C., Floreano, D., Fua, P., and Gervaix, F. (2011). The accuracy of automatic photogrammetric techniques on ultra-light UAV imagery. In Proceedings of the International Conference on Unmanned Aerial Vebicle in Geomatics (UAV-g), Zurich, Switzerland. ISPRS Archives - Volume XXXVIII-1/C22, p. 125-130.

LOWE, D. G. (2004). Distinctive image features from scale-invariant keypoints, International Journal of Computer Vision 60: p. 91-110.

PIERROT-DeSEILligNY, M. 2012. MicMac, Apero, Pastis and Other Beverages in a Nutshell!. Disponível online http://logiciels.ign.fr/?Micmac. Acedido em 2014-06-16

PIERROT-DESEILligNY, M., and Clery, I. (2011). Apero, an open source bundle adjusment software for automatic calibration and orientation of set of images. In Proceedings of the ISPRS Symposium, 3DARCH11, p. 269-277.

PIERROT-DESEILligNY, M., and Paparoditis, N. (2006). A multiresolution and optimization-based image matching approach: An application to surface reconstruction from SPOT5-HRS stereo imagery. In ISPRS Workshop on Topographic Mapping From Space (With Special Emphasis on Small Satellites) Vol. 36 (Part 1/W41), Ankara, Turkey

ROY, S., and Cox, I.J. (1998). A maximum-flow formulation of the N-camera stereo correspondence problem. In Computer Vision, 1998. Sixth International Conference on, p. 492-499.

SenseFly 2014. The swinglet CAM. Disponível online http://www.sensefly.com/. Acedido em 2014-06-16.

SKARLATOS, D. (1999). Orthophotograph Production in Urban Areas, The Photogrammetric Record 16 : 643-650. 
TURNER, D.; Lucieer, A. \& Watson, C. (2012). An Automated Technique for Generating Georectified Mosaics from Ultra-High Resolution Unmanned Aerial Vebicle (UAV) Imagery, Based on Structure from Motion (SfM) Point Clouds, Remote Sensing 4 : 1392-1410.

WATTS, A. C.; Ambrosia, V. G. \& Hinkley, E. A. (2012). Unmanned aircraft systems in remote sensing and scientific research: Classification and considerations of use, Remote Sensing 4 : 1671-1692.

ZHOU, G.; Chen, W.; Kelmelis, J. \& Zhang, D. (2005). A Comprehensive Study on Urban True Orthorectification, IEEE Transactions on Geoscience and Remote Sensing, 43: 2138-2147. 
Série Documentos

Imprensa da Universidade de Coimbra

Coimbra University Press

2015

- U M

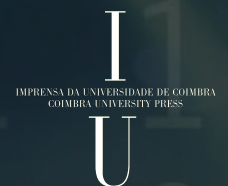

